\title{
Lessons learnt from a genetic disease registry in Hong Kong
}

\author{
Stephen TS Lam ${ }^{1}$ *, MD, FHKAM (Paediatrics), CH To², PhD, KW Leung ${ }^{2}$, FCOphth HK, FRCOphth, SP Yip 3 , PhD, \\ Ivan FM Lo ${ }^{4}$, FHKCPaed, FHKAM (Paediatrics), KP Tsang ${ }^{5}$ \\ ${ }^{1}$ Department of Clinical Genetics Service, Hong Kong Sanatorium \& Hospital, Hong Kong \\ ${ }^{2}$ School of Optometry, The Hong Kong Polytechnic University, Hong Kong \\ ${ }^{3}$ Department of Health Technology and Informatics, The Hong Kong Polytechnic University, Hong Kong \\ ${ }^{4}$ Clinical Genetic Service, Department of Health, Hong Kong \\ ${ }^{5}$ Retina Hong Kong, Hong Kong \\ *Corresponding author: taksumlam@gmail.com
}

\section{Purpose of a genetic disease registry}

A registry for a specific clinical condition serves multiple purposes. Firstly, it provides a systemic index of patients and families with the condition. Secondly, it helps in the classification and management of patients with the condition. Thirdly, it is a good resource for research into the epidemiology, phenotype and genotype variations, disease mechanisms, and treatment modalities of the condition. Fourthly, from the above functions, it works as a tool for advocacy for the welfare of patients with the condition. To serve these purposes for patients with retinitis pigmentosa (RP) in Hong Kong, a registry was started, and the following is a summary of the issues encountered and lessons learnt.

\section{Retinitis pigmentosa registry in Hong Kong}

A rare genetic eye disorder, RP causes progressive visual loss due to degeneration of cells in the retina, estimated to affect 1 in 4000 people in the United States and worldwide. ${ }^{1}$ There are, however, no reported prevalence data in Hong Kong. The diagnosis of RP is based on the clinical features which include symptoms of night vision impairment and tunnel vision, and the findings of typical retinal appearance of attenuated blood vessels, so-called bone spicule pigmentation or its variant appearance and an atrophic optic disc. These are supported by an abnormal constricted visual field and a nonrecordable or abnormal electroretinography with diminished $a$ and $b$ waves with increased implicit time. The RP is also highly genetically heterogeneous, with more than 80 genes implicated and their mode of inheritance varies. This means one patient's RP may be a different genetic disorder from that of another RP patient, with different inheritance, natural course, and prognosis. The rarity of the disease and the associated genetic heterogeneity are major obstacles to facilitating research and enhancing medical care to these patients and their families. The Hong Kong Retinitis Pigmentosa Society was started in 1995 as a patient group, and subsequently renamed as Retina Hong Kong. Under the auspices of Retina Hong Kong, a Scientific and Medical Advisory Committee (SMAC) was established. In 1997, the SMAC secured a grant from the Healthcare and Promotion Fund of the Hong Kong Government to start organising the Hong Kong Patients Registry of Retinal Degenerations. It was the original intention for this registry to cover most retinal degenerative conditions. However, because of resource constraints, it was decided that the scope should be limited to RP as the first group of members to be covered. Hence, the registry was also abbreviated as the Hong Kong Retinitis Pigmentosa Registry. It turned out to be the first registry of its kind among Chinese populations in the whole world, and to the best of our knowledge, there is no other Chinese Registry presently.

The objectives of the Retinitis Pigmentosa Registry are three-fold, including:

- To provide detailed ophthalmic and genetic examinations for inherited retinal degenerative diseases patients so as to ascertain the symptoms and the genetic pattern of the patients.

- To collect relevant data and build up a database for the benefit of future scientific, medical, and sociological researches.

- To provide lifelong follow-up services for patients who participate in the project and to notify and refer them for appropriate treatment as soon as it is available.

The holder of the Registry is the Retina Hong Kong, whose members were offered the option of voluntary participation in the Registry. Patient members were invited to be seen by the specialists in the SMAC in a sequence as outlined in the flowchart in the Figure for clinical examinations, diagnostic testing, and counselling. 
Right at the start of their participation, members were advised of the detailed flow of the whole process. More importantly, they were advised of their rights in this participation before signing for consent. Their rights included:

- To obtain detailed ophthalmic examination and eye care apart from advice on genetics.

- To know well their condition and progress through different clinical examinations.

- To be informed of all findings that might help in their treatment plan.

- To make use of relevant information to formulate their future plan on career and family.

- The Registry is maintained strictly in accordance with the Personal Data (Privacy) Ordinance.

- Participants may obtain all information relating to their own case, which is stored in the database of the Registry.

To ensure that participants are well informed of the flow of the registry and their rights and obligations, pamphlets were designed and made available as part of the informed consent process.

The first clinical assessment for the participants was an optometric evaluation at The Hong Kong Polytechnic University (HKPU), where refraction studies, ocular health assessment, visual field test, electroretinogram studies, and fundus photography were performed. This was followed by an ophthalmologic examination with dilated fundus evaluation. As shown in the Figure, eye diseases are defined upon clinical criteria as to whether they fall into the categories of known hereditary nature.

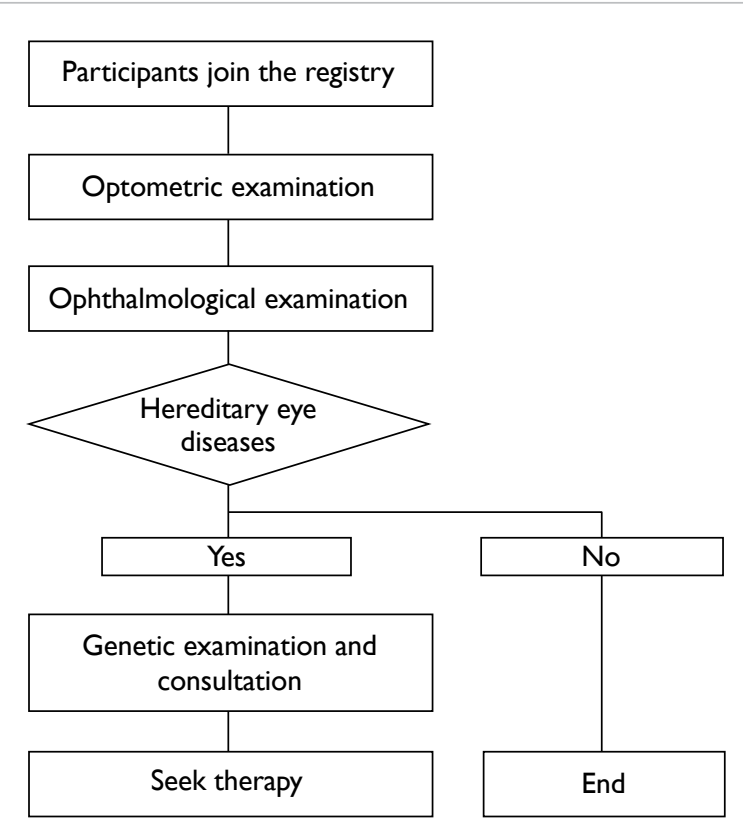

FIG. Flowchart of the retinitis pigmentosa registry
The third clinical assessment was genetic evaluation and counselling together with sample collection (blood sample with DNA extraction) performed at the Clinical Genetic Service of the Department of Health. Further genetic testing was carried out at the molecular laboratory of HKPU for mutational analysis. Periodic clinical and molecular conferences were conducted among members of the SMAC to confirm the exact clinical diagnosis, genetic defects, and inheritance patterns for individual participants to guide further genetic counselling, whence other family members were also invited to participate on a voluntary basis.

Periodic assessment of the Registry was conducted by the SMAC and reported to the Retina Hong Kong. The first of these reports covered the period 1999 to 2005, during which a total of 94 families were documented. After detailed evaluation was conducted conjointly by the specialists in the SMAC, the diagnosis of RP was documented in 72 of these families with inheritance pattern classified as simplex (44,61.1\%), autosomal dominant (12, 16.7\%), autosomal recessive $(12,16.7 \%)$ and X-linked recessive (4, 5.6\%), which showed comparable results to those published by other centres $^{2,3}$; and the remaining 22 families of alternative diagnosis with various genetic backgrounds. It was noted that the phenotype of patients with non-RP retinal degenerations may overlap with that of RP, leading to erroneous clinical diagnosis, as exemplified in $30 \%$ of this cohort. By combining clinical data and genetic analysis, a more definitive diagnosis could be achieved.

Cases of RP and other retinal degenerative diseases were categorised and published. ${ }^{4,5}$ In these early days of molecular analysis, genetic studies were carried out on putative genes individually, hence on a gene by gene basis, instead of on a multiplex basis. As expected, the positive yield of such a laborious approach was necessarily low.

\section{Funding issues}

It was most fortunate that the Healthcare Promotion Fund has sponsored this registry for nearly 5 years (1997-2002). With the cessation of this sponsorship, the Registry could not be sustained, and was suspended in 2002. It was resumed in April 2003 after further sponsorship was obtained from Retina Hong Kong, with additional financial input from the individual institutes to which the members of SMAC were affiliated.

\section{Further progress of the registry}

With the advent of the massively parallel sequencing (also known as next generation sequencing) technology, it became possible for mutational analysis to be performed on a multiplex instead of 
single gene basis. The previous cohort examined until 2002 was expanded with new entries to a total of 116 families by 2012 . Their samples were subjected to next generating sequencing performed on MiSeq (Illumina) system, initially at HKPU, and, as an ongoing process, also in Clinical Genetic Service of the Department of Health. Additional mutations were identified and put to good use in genetic counselling of the affected individuals and families.

\section{Lessons learnt}

The above narration has highlighted some issues pertaining to the success or failure of this kind of registries. The Retinitis Pigmentosa Registry is notably the first of its kind among all Chinese populations, and hence has set an exemplary function for others. The unique feature of the Retinitis Pigmentosa Registry is that it is initiated and led by a patient organisation, which works for the welfare of its members. As such, the interest of the patients and their families were considered the primary objective of the registry. All necessary steps were taken to upkeep the ethical principles for patient confidentiality and informed consent as exercised in the design and implementation of this registry. In addition, a central core of highly motivated individuals and institutes from the clinical and scientific community is needed to provide the academic and service input to make it viable. From this combined clinical and scientific approach, an accurate and reliable diagnosis could be provided for genetic counselling of many subjects. The data attained from the studies had also provided information for the spectrum of retinal degenerations seen in Chinese populations.

Yet, this registry has also uncovered a major problem with setting up genetic registries: financial instability. The experience shown here is that a strategy is needed to ensure the financial sustainability of the project. To achieve the objectives of the provision of ongoing care for the participants, a secure source of funding is needed on a long-term basis. It is advisable to explore different sources of financial commitment both in the public and private sectors.

\section{Author contributions}

All authors contributed to the concept or design of the study, acquisition of data, analysis or interpretation of data, drafting of the manuscript, and critical revision of the manuscript for important intellectual content. All authors had full access to the data, contributed to the study, approved the final version for publication, and take responsibility for its accuracy and integrity.

\section{Conflicts of interest}

The authors have no conflicts of interest to disclose.

\section{Funding/support}

The funders mentioned in the article had no role in study design, data collection/analysis/interpretation or manuscript preparation.

\section{References}

1. National Eye Institute. Retinitis pigmentosa. Available from: https://www.nei.nih.gov/learn-about-eye-health/ eye-conditions-and-diseases/retinitis-pigmentosa. Accessed 1 Jan 2021.

2. Ferrari S, Di Iorio E, Barbaro V, Ponzin D, Sorrentino FS, Parmeggiani F. Retinitis pigmentosa: genes and disease mechanisms. Curr Genomics 2011;12:238-49.

3. National Center for Advancing Translational Sciences: Genetic and Rare Diseases Information Center. About GARD. Available from: http://rarediseases.info.nih.gov. Accessed 1 Jan 2021.

4. Yip SP, Cheung TS, Chu MY, et al. Novel truncating mutations of the CHM gene in Chinese patients with choroideremia. Mol Vis 2007;13:2183-93.

5. Lim KP, Yip SP, Cheung SC, Leung KW, Lam ST, To CH. Novel PRPF31 and PRPH2 mutations and co-occurrence of PRPF31 and RHO mutations in Chinese patients with retinitis pigmentosa. Arch Ophthalmol 2009;127:784-90. 